\title{
Creando puentes entre la formación y la creatividad: Una experiencia de investigación activista feminista ${ }^{1}$
}

\author{
Karina Fulladosa-Leal ${ }^{2}$ \\ Universidad Autónoma de Barcelona, Barcelona, España ${ }^{3}$ \\ karinafulladosa@hotmail.com \\ Recibido: 2 de octubre de 2013 \\ Aceptado: 24 de enero 2014
}
Agradezco a Sindihogar por permitirme acompañarlas, a Norma Falconi, por contagiarme su pasión y por compartir sus conocimientos, a todo el grupo FIC, a mi tutora Dra. Marisela Montenegro por sus comentarios y finalmente a mis amigas, activistas, académicas y hermosas lectoras Daniela Osorio e Itziar Gandarias. Este trabajo se presenta como artículo de investigación científica, forma parte del proyecto de tesis titulado "Mujeres en movimiento: ampliando los márgenes de participación social y política en la acción colectiva como trabajadoras del hogar y el cuidado", subscrita al Departamento de Psicología Social de la Universidad Autónoma de Barcelona. Se financió en el marco del Proyecto "Instalación de la Unidad de Posgrados: Consolidación y Fortalecimiento de la Investigación en Psicología y de los Posgrados de la Facultad de Psicología de la Universidad de la República de Uruguay", a través de la AECID.

2 Magister en Investigación en Psicología Social de la UAB. La última publicación se refiere a "Una aproximación a los procesos de subjetivación de las trabajadoras del hogar y el cuidado sindicalizadas".

3 Estudiante de Doctorado en el Departamento de Psicología Social de la UAB y miembro del grupo Fractalidades en Investigación Crítica (FIC). 


\title{
Creando puentes entre la formación y la creatividad: Una experiencia de investigación activista feminista
}

\section{Resumen}

El presente artículo aborda la experiencia de formación realizada en el sindicato de trabajadoras del hogar y el cuidado (Sindihogar) en Barcelona a través de un trabajo colectivo. Para ello, realizo una reflexión articulando la propuesta de los conocimientos situados (Haraway, 1995/1991) y la investigación activista feminista (Biglia, 2005) las cuales me han permitido tender un puente que transite desde las reflexiones sobre el conocimiento y la cotidianidad donde estos se producen. El escrito se presenta en una primera parte expositiva sobre Sindihogar. Luego se plantea un apartado metodológico que guió el proceso y la puesta en práctica. Finaliza con algunas reflexiones a considerar en torno a la investigación activista feminista.

Palabras clave: investigación activista feminista; Conocimientos situados; Sindicato de trabajadoras del hogar y el cuidado

\section{Creating Bridges between Training and Creativity: A Feminist Activist Research Experience}

\begin{abstract}
This article deals with the training experience performed in the home and care worker syndicate (Sindihogar) in Barcelona, by means of a collective work. For this purpose, I write a reflection joining the concept of situated knowledges (Haraway, 1995/1991) and the feminist activist research (Biglia, 2005). These concepts allowed me to lay a bridge from the reflections about knowledge and the everyday circumstances where it is produced. The first part of this work is presented as an exposition about Sindihogar. Then, the methodological section that guided the process and the implementation is explained. It concludes with some reflections related to feminist activist research to be considered.
\end{abstract}

Keywords: feminist activist research; situated knowledges; home and care worker syndicate

\section{Criando pontes entre a formação e a criatividade: Uma experiência de pesquisa ativista feminista}

\section{Resumo}

O presente artigo discute a experiência de educação realizada no sindicato de trabalhadoras da moradia e do cuidado (Sindihogar) em Barcelona através de trabalho coletivo. Para isso, realizo uma reflexão que articula a proposta dos conhecimentos situados (Haraway, 1995/1991) e a pesquisa ativista feminista (Biglia, 2005) as quais permitiram-me tender uma ponte que transita desde as reflexões sobre o conhecimento para a cotidianidade onde estes ocorrem. O texto apresenta-se em uma primeira parte expositiva sobre Sindihogar. Posteriormente se levanta um apartado metodológico que guiou o processo e a implementação. Finaliza com algumas reflexões para considerar em torno da pesquisa ativista feminista.

Palavras-chave: pesquisa ativista feminista; conhecimentos situados; sindicato de trabalhadoras da moradia e o cuidado 


\section{Introducción ${ }^{4}$}

La producción de conocimientos es una práctica social y por tanto es necesario plantear la producción de los mismos a través de las re-conceptualizaciones, tanto dentro del ámbito académico como afuera de este (Adán, 2006) para generar diálogos compartidos en torno a ellos. De esta manera, es crucial entender que no es posible establecer ningún espacio -vital o discursivo- que quede por fuera del influjo de la sociedad, lo que en este escrito me ha llevado a revisar las criticas desde los feminismos a las ciencias clásicas con sus postulados de neutralidad en el conocimiento, el valor de la objetividad y la propia configuración objeto/sujeto.

Es decir que es necesario sortear ciertos desafios dentro del universo académico, como es la búsqueda de distanciamiento de lo que producimos, un lenguaje especializado y el desconocimiento de las relaciones de poder que se encuentran imbricadas en los procesos en los cuales participamos. Así, me he resituado en este escenario para dialogar con otras autoras que han desmitificado los supuestos de neutralidad y universalidad del conocimiento que las ciencias han pujado por sostener y comenzar un recorrido hacia los saberes compartidos.

En esta línea, la reflexión feminista ha aportado una visión del conocimiento como práctica social desde donde poder recrear procesos de investigación hilando reflexión y práctica. En este sentido, una de sus referentes dentro del empirismo feminista, Longino (1990) sugiere que la estrategia feminista pasa por elaborar el conocimiento desde una práctica feminista, es decir, generar nuevos sistemas de valores, capaces de incidir dentro de las propias comunidades científicas.

Por otra parte, Haraway nos propone dar un paso más con respecto a la comprensión o cambio de valores. Las posibilidades de transformación del conocimiento por parte de las feministas (Adán, 2006) no pasa tanto por destacar las voces de las mujeres, como por las nuevas articulaciones en la red del conocimiento-politica-cultura-naturaleza, lo que ella llama tecnociencia en la que las mujeres estamos inmersas

\footnotetext{
Nota para los y las lectoras: luego de tener que tomar una decisión para escribir este artículo en singular o plural, comentario que fue realizado tanto por los revisores como por mí tutora, me encontré con este texto que de alguna manera pone palabras a algunos de los conflictos por los cuales atravesaba al narrar la experiencia: "A veces, nos expresamos en singular, otras en plural, y jugamos a desdibujar los géneros no para desorientar a quien nos lea, sino para compartir las contradicciones que habitamos y los lugares desde dónde nos situamos, a fin de evidenciar algunos de los múltiples matices que contienen las prácticas de investigación activista" (Pantera R., 2004, p.191)
} 
(Adán, 2006). Para ello, acuña el concepto de los conocimientos situados como forma de evidenciar el lugar desde donde parten las investigadoras, sus posiciones y posturas politicas, asumiendo que no hay un acto neutral, sino una visión del mundo de la cual se toma partido con las consecuencias que eso entraña.

El hilo conductor que propongo entonces, tiene que ver con la idea común de los feminismos polifónicos, globales o multiculturales en torno a la necesidad de que los feminismos -como teoria y práctica- no reproduzcan las formas de los discursos hegemónicos patriarcales que han tendido a la uniformidad y a la homogenización de las realidades de las mujeres (Adán, 2006), sino hacia la producción de conocimientos colectivos (en particular academia-movimientos sociales, académico/activista/militante) que permitan confrontar las formas de poder subordinante que se instalan en el saber disciplinar, las políticas públicas y los patrones de valor cultural, a partir de un pensamiento que hace crítica desde la experiencia y los modos de existencia.

Teniendo en cuenta el amplio recorrido y esfuerzos de los feminismos, por abordar y complejizar la producción de conocimiento, me he aproximado a la investigación activista feminista (en adelante IACF), entendiendo que es una apuesta política para situarnos en nuevas formas de conocer, pensar y actuar, acompañando procesos de transformación social. El compromiso asumido al co-participar en la conformación sindical de las Trabajadoras del Hogar y el Cuidado en Catalunya (Sindihogar) se basa en los supuestos orientadores de la IACF que parten más allá de las elecciones de las metodologías especificas (cualitativo, cuantitativo, entrevista, observación etc...) y le atribuyen igual importancia a los fundamentos ontológicos y epistemológicos que sirven de base a la producción de conocimientos.

La articulación entre los conocimientos situados (Haraway, 1995/1991) y la IACF (Biglia, 2005) supone la posibilidad de transitar hacia la producción de conocimiento a través de la experiencia de las diferentes subjetividades y crear una base para la lucha colectiva, tendiendo puentes entre el espacio académico y el activismo. Dicho de otro modo, y llevándolo al plano concreto, a través de la experiencia de la autoformación dentro del Sindicato de Trabajadoras del Hogar y Cuidado, planteo una reflexión sobre la apuesta política de dicha metodología y el trabajo realizado entre todas.

En los siguientes apartados dialogo sobre las divergencias de la IACF con la IA(P) y su convergencia con los conocimientos situados, luego introduzco la historia y lugares compartidos en Sindihogar 
donde se gestó la formación que contextualizan la experiencia, articulándolo con la apuesta metodológica. Posteriormente presento las reflexiones sobre las potencialidades y limitaciones de la IACF, finalizando con algunas consideraciones sobre todo el proceso.

\section{La IACF: divergencias con la IA (P) y convergencias con los conocimientos situados}

La investigación activista feminista (Biglia, 2005) parte de repensar la metodología y las técnicas de investigación, sobre todo las referentes a la investigación-acción y la investigación-acción-participante, así como de redefinir las formas epistémicas y las ontologias que nutren la investigación psicosocial.

En este sentido, la propuesta partió de revisitar la investigación acción (IA) (Lewin, 1946) como la investigación acción participativa (IAP) (Fals Borda, 1993) reflexionando a partir de su puesta en práctica y articulándose con otra forma de hacer. Por lo cual, es necesario plantear las divergencias que Biglia (2005) remarca con respecto a estas dos metodologías mencionadas, además de algunas consideraciones realizadas a partir de la puesta en práctica y su conexión con los conocimientos situados.

Comienzo por exponer algunas divergencias que la IACF plantea con relación a la IA y la IAP. En primer lugar, se plantea una forma diferente de entender la acción para el cambio social. En este sentido, la IA nos propone que este cambio social se produzca y vaya más allá de la investigación. Sin embargo la IACF parte de la idea de que los conocimientos auto-reflexivos dentro de un colectivo social ya son parte del cambio (Biglia, 2005). Nuestra presencia en el campo-tema (Spink, 2003) ya nos plantea determinadas consecuencias: "El campo-tema no es un acuario que miramos del otro lado del vidrio; es algo de lo que formamos parte desde el primer momento que decimos: 'estoy trabajando con..."5 (Spink, 2003, p.36).

Esto implica que desde el momento que nos planteamos el campo, la pregunta y los trazos que guiarán la investigación, ya estamos dialogando con otras y pensando cómo podemos ser útiles o contribuir con ese tema con el cual nos sentimos comprometidas.

5 Texto original: "O campo-tema não é um aquário que olhamos do outro lado do vidro; é algo do qual fazemos parte desde o primeiro momento em que dissemos, "estou trabalhando com"..." (Spink, 2003, p. 36), traducción de la autora. 
En segundo lugar, la IA plantea la colaboración directa entre investigadora(s) y la colectividad, haciendo necesario en ocasiones cursos de formación para la implementación del diseño. En IACF los planteamientos se realizan de forma más flexible (Biglia, 2005), de hecho en el curso de esta investigación el diseño, como los objetivos los he planteado de forma dialógica, es decir que si bien partían de mis reflexiones a través de diferentes lecturas respecto al tema, se han compartido en diferentes espacios con las activistas de Sindihogar y otras investigadoras e investigadores generando un espiral que nutría y retroalimentaba una mirada inicial.

Un tercer punto, es que muchos de los trabajos de IA como de IAP (especialmente) son realizados porque existe una comunidad interesada en desarrollar dicho proceso de investigación. En caso de existir algún tipo de concientización esta será producto de un proceso no solo de investigación sino de todo el colectivo. La IACF no pretende concienciar a las participantes en tanto entiende que las personas pueden darse cuenta de lo que les pasa y dentro de las posibilidades que disponen y eligen la que sea más conveniente a su situación (Biglia, 2005).

Esta posición es cercana a la idea de Hill Collins (2000) quien expresa que los saberes colectivos de las mujeres negras desafian dos interpretaciones sobre la conciencia de los grupos oprimidos, que se pueden conectar con los saberes de las trabajadoras del hogar y el cuidado que participan de Sindihogar. Una que afirma que los grupos subordinados se identifican con el grupo dominante y no tienen una interpretación válida independiente de su propia opresión. La segunda, que los oprimidos son menos persona y por lo tanto menos capaces de interpretar sus experiencias. Ambas explicaciones intentan sugerir la presunta falta de activismo político por su propia conciencia de subordinación. Sin embargo, lo que debemos reflexionar es que, cualquier conciencia independiente expresada por los grupos oprimidos es visualizada como si no fuera de su propia creación o como si fuera inferior a la de los grupos dominantes (Hill Collins, 2000) y es por ello que se producen estos saberes en los márgenes. En ningún caso se concientiza sobre la práctica feminista ni sobre las diferentes opresiones dentro del trabajo del hogar, sino que planteábamos la posición de la investigación y nos preguntábamos si podiamos encontrar conexiones para llevarla a cabo.

Un cuarto punto hace referencia a la devolución y difusión mencionadas en la IAP, donde el trabajo de Callén et al. (2007) nos sugiere cuestionar una posición de uni-direccionalidad privilegiada desde los 
sujetos hacia los objetos de investigación. En este sentido, la IACF propone formas de conocimiento no propietarias y re-inventar espacios de acceso libre (plataformas virtuales, revistas de acceso abierto, revistas desde los movimientos sociales) donde los textos puedan circular, sin la marca copyright.

Por otro lado, contamos con la advertencia que algunas y algunos autores (IOE, 1993) hacen respecto a la posibilidad de que el discurso de la participación en la IAP pueda ser coaptado desde organismos oficiales para legitimar acciones que no van de la mano con los intereses de las personas afectadas, "ya que la metodología de la IAP (especialmente si se lleva a una tecnificación de la misma, alejada de su potencial político) puede ser utilizada con fines de normalización y adaptación social" (Montenegro, 2001, p. 1999). En este sentido, remarcar su componente feminista en la IACF tiene como objetivo configurarse como un componente de acción política:

[...] se quiere apelar a una noción de política que traspase la idea de lo organizado, de lo ideológico y de lo racional, para extenderla al espacio de lo cotidiano, de la interacción dialógica con los otros, y de la acción". (Pujal, 2003, p.131)

En última instancia, esta se aleja de los presupuestos clásicos de la IAP, donde se realiza una planificación, ejecución y evaluación. En la puesta en práctica de dicha metodología, podemos dejar espacio a la apertura e incertidumbre, nos permitimos que los acontecimientos nos lleven a construcciones más libertarias y desordenadas, porque parten del trabajo conjunto (León, 2010). La investigación se realiza mediante la identificación de un punto de partida desde el cual se estaría "caminando sin saber muy bien cómo y a donde se llegará" (Spink, 2003), lo cual no lo aleja de una postura ético-política, sino que esta dimensión está en el encuentro y las posibles conexiones que en este caso se producen con las activistas.

En cuanto a los aportes que nos brinda el núcleo de la propuesta de Haraway, lo tomo de las palabras de Araiza (2009) que nos habla del amor y el contacto que surge con relación a lo que investigamos. En este sentido nos propone la mezcla de nuestros amores privados con las pasiones teóricas, es decir, qué implica conocer, cómo se conoce en el plano académico y qué es aquello que se acerca más a 
nuestras vidas cotidianas ${ }^{6}$. O reconocer que la manera académica de conocer es parte de nuestra cotidianidad. (Araiza, 2009).

Es decir que parte de nuestra objetividad encarnada transita por entender:

[...] que mi problema y "nuestro" problema es cómo lograr simultáneamente una visión de la contingencia histórica radical para todas las afirmaciones del conocimiento y los sujetos conocedores, una práctica crítica capaz de reconocer nuestras propias "tecnologías somáticas" para lograr significados y un compromiso consentido que consiga versiones fidedignas de un mundo "real", que pueda ser parcialmente compartido y que sea favorable a los proyectos globales de libertad finita, de abundancia material adecuada, de modesto significado en el sufrimiento y de felicidad limitada. (Haraway, 1995/1991, p. 321)

Lo que nos invita a arriesgarnos a una investigación viva (término adoptado por una activista, en CC. 29/09/137) sorteando el paradigma occidental sobre una objetividad planteada en la emblemática separación entre mente y cuerpo, de la distancia y de la responsabilidad neutral. Sin embargo, a través de este recorrido apuesto por:

[una] objetividad feminista [que] trata de la localización limitada y del conocimiento situado, no de la trascendencia y el desdoblamiento del sujeto y el objeto. Caso de lograrlos, podremos responder de lo que hemos aprendido y de cómo miramos. (Haraway, 1995/1991, p.327)

En este sentido, la IACF comparte su base epistémica (que a lo largo del texto iré entrelazando) a partir de considerar una posición situada, parcial y con(sentido) a la responsabilidad de lo que generamos y producimos. Así, nos encontramos en relación con otras, donde podemos crear una complicidad a partir también de compartir nuestra historia y vivencia, politizando nuestra vida con las activistas en un espacio que nos reúne con un objetivo común por la defensa de los derechos de las trabajadoras del hogar y el cuidado. Esto nos permite

6 Podemos comprender la vida cotidiana "[...] como espacio en el que se entretejen relaciones sociales y compromisos, se configuran y confrontan lealtades, se despolitizan y politizan problemas, se separa y "reunifica" vida y política, en otros términos se traban los procesos de hegemonía y se despliegan múltiples procesos de resistencia” (Grimberg, 2009, p. 92).

7 Extractos del cuaderno de campo, en adelante CC. con iniciales y fecha. 
transformarnos y aproximarnos a otras formas de ser mujeres, trabajadoras activistas, académicas porque como dice Jackeline "el cambio tiene que empezar por casa” (activista, en CC. 21/06/13).

\section{Historia y lugares compartidos: articulándonos en la La formación}

A modo de presentación del colectivo, Sindihogar se conforma en diciembre de 2011 como el primer sindicato independiente de trabajadoras del hogar y el cuidado en Cataluña. El sindicato está influido por múltiples experiencias asociacionistas de las cuales las trabajadoras eran parte, lo que ha devenido en una organización de base sindical que tiene como objetivo reivindicar sus derechos como trabajadoras y denunciar las condiciones de trabajo dentro de la precariedad económica (economía sumergida, bajos salarios, falta de cobertura por desempleo, entre otras), política (escaso nivel de organización, dificultades para la negociación ante sus empleadores y empleadoras y otros actores/actrices sociales, etc.) y social (desvalorización e infravaloración de su trabajo, discriminación por raza/etnia, genero, sexualidad, etc.).

La decisión de poder trabajar conjuntamente con el sindicato surge de un primer acercamiento a través de un trabajo realizado en el 2011. Esta elección buscaba comprender su potencial politico en la reivindicación y dialogo sobre lo que son los trabajos del hogar y del cuidado, su organización en lo social y las problemáticas que se encuentran entrelazadas con los mismos, así como por ser el único sindicato de trabajadoras del hogar dentro del Estado español.

Me involucré en el sindicato en enero de 2012 al proponer una investigación de producción de conocimiento conjunto y colaborativo. La inquietud por conocer la forma de organización, acompañar y apoyar este proceso fue planteada en una asamblea con más de veinte trabajadoras y diversas mujeres que provenian de colectivos de mujeres migrantes. Desde ese momento comencé a participar de forma activa, acompañando y colaborando en la consolidación de la organización, lo que derivó en una forma de hacer próxima a la IACF.

Durante el primer año, además de participar en asambleas, manifestaciones y jornadas, realicé cuatro narrativas utilizando la técnica de las producciones narrativas (Balasch y Montenegro, 2003) con activistas del sindicato. Aquel proceso abrió el camino para el inicio de una IACF. Las acciones que realizábamos en el sindicato ya eran 
parte del proceso de investigación, ya que me encontraba implicada en las mismas y actuando en ese espacio, por ello me propuse seguir los itinerarios y trayectorias del colectivo y ver si de las acciones conjuntas podía surgir un relato o narración que diera cuenta de este recorrido. Así, me situé asumiendo ser parte del colectivo, permeando el trabajo desde la propia subjetividad sin una actitud neutral y parcial, sino afin con la práctica activista desarrollada, habitando un espacio que Biglia y Zavos llaman de bordeline (2005), como activista desde un rol de investigadora académica.

La autoformación dentro del sindicato hace parte de los diálogos mantenidos en las asambleas por una inquietud que nos atravesaba y tiene que ver con una visión compartida en cuanto a la falta de información e interpretación de las normativas del trabajo del hogar, sus continuas modificaciones desde enero de 2011 y la posibilidad de discutir entre el colectivo y con otras las miradas sobre el trabajo de cuidados, el género y las diferentes violencias que se entretejen en la vidas de todas (económicas, políticas, sociales, machistas).

De esta manera, junto con otra compañera comenzamos a diseñar el formato y los temas para luego discutirlos entre todas. Esto nos llevó a que se movilizaran una serie de agencias que tuvieron que ver con: encontrar un espacio propicio para llevar adelante la actividad, realizar la convocatoria de las ponentes que llevarian adelante los talleres, de las compañeras que realizarían las actividades artísticas y la organización del catering para el fin de la jornada. Esto propició un trabajo en red donde cada una podía estar -en la medida de sus posibilidades, espacio-tiempo e intereses-, lo cual produjo diferentes participaciones que se iban intercambiando. Algunas activistas presentaron propuestas artísticas, otras realizaban las presentaciones de las ponentes, algunas nos encargamos del diseño y la presentación de algunos temas, valiendo los recursos y herramientas de cada una.

El objetivo principal entonces fue trabajar los temas que más impacto tienen para las trabajadoras del hogar y el cuidado en el Estado Español. Esto sin dejar de lado el tiempo para la creatividad y la re-creación, teniendo en cuenta que los encuentros se realizarian los sábados en la tarde, luego de toda una semana laboral. No solo se planteaba como un espacio de reflexión imperante sino también de disfrute y movimiento.

Esta forma de hacer -construyendo espacios en los que se puedan producir conocimientos y acciones compartidas- partió de una visión crítica respecto a la tendencia tanto de los organismos 
públicos, organizaciones civiles y asociaciones de plantear formaciones hacia el colectivo de mujeres trabajadoras del hogar y no con las trabajadoras, desde una posición unidireccional y sin tener en cuenta las necesidades concretas que tienen como colectivo.

Así las cosas, nos involucramos en la ampliación del conocimiento de las normativas (laborales, nacionales e internacionales) y temas sugeridos (cuidados, género, migración, violencia), como también en la construcción de un espacio donde circulara una red de afectos, contención y soporte, donde la experiencia y las diversas situaciones pudieran ser narradas. La formación se convirtió en una herramienta que nos articuló y posibilitó establecer diálogos entre diferentes colectivos y personas involucradas tanto al mundo académico como activista.

Los tres ejes y las actividades que se propusieron consistieron en:

(i) Conocimiento y reflexión sobre las normativas que regulan el trabajo del hogar y el cuidado (Convenio 189 de la OIT y el Decreto 1620/2011 y su posterior modificación), a cargo de la antropóloga Gabriela Poblet, activista de Papeles para Todas Ilundi Patraquim; la activista de Sindihogar y Papeles para Todas, Norma Falconi; la investigadora y activista en Sindihogar, Karina Fulladosa; la poesía de Gloria Rivas y la música Góspel de Rebeca Iyabode (activistas de Sindihogar) sumándose el coro que acompañó desde las participantes. Ese día al final de la jornada, Rebeca también leyó un comunicado y realizó una oración por la muerte a causa de la violencia machista de dos mujeres en Bilbao, provenientes de Colombia y Nigeria.

(ii) Migración y trabajo del hogar y cuidado, qué relación existe entre la mujer migrada y el CIE (Centro de Internamiento de Extranjeros), la irregularidad sobrevenida en tiempos de crisis, trabajadoras del hogar sin papeles expuestas a la expulsión, la reformulación de cruces indispensables entre igualdad, politicas de extranjería y trabajo. Estas actividades estuvieron a cargo del COL.LECTIU EINES finalizando con una performance que dirigió Denys Blacker.

(iii) Género y cuidados. Dentro de la temática de cuidados se abordo qué entendemos por cuidados, la globalización de los cuidados, el derecho a recibir cuidados, el derecho a elegir cuidar o no cuidar y a condiciones laborales justas, el bienestar para las personas cuidadas y las cuidadoras, invitando a la activista e investigadora Silvia López Gil. Dicha actividad finalizó con la muestra de Danza Nepalí. Dentro de la temática de género se abordaron las diferentes violencias: racial, 
psicológica (chantaje emocional, culpa, cadena de favores, deuda) y las posibles estrategias que presentó la activista feminista y antimilitarista Monsterrat Cervera. El cierre de la actividad se realizó con un espacio de danza y movimiento a través de un grupo musical multicultural Red Ras y los amigos del alcalde.

Las elecciones respecto a las personas que participaron de diversas formas en las jornadas partieron de tres inquietudes. En primer lugar, con relación a las ponentes que fueran afines a las temáticas a abordar, no solo en un plano teórico sino también que participaran activamente de los movimientos sociales. En segundo lugar, contar con la red que cada una tuviera en su entorno y siguiera este propósito. Y por último, crear un espacio donde acercarnos a través de nuestros gustos musicales, culturales y de ocio. Así, algunas activistas compartieron su escritura, otras su voz, la danza y también se abrió la posibilidad de invitar a grupos musicales y personas relacionadas con el performance que -como mencionaba una activista- no es más que otra forma de descubrir-nos, conocer-nos y saber que "los inmigrantes también podemos hacer performance" (N. activista, en CC. 22/06/2013).

Un aspecto no menor fue el espacio donde se desarrollaron las jornadas todos los sábados de junio de 2013, en el Centro Cultural Francesca Bonnemaison. Esta institución emblemática fue creada por Francesca Bonnemaison en el año 1909 junto con otras mujeres y fue la primera Biblioteca Popular para la Mujer en Europa. Se acercó de esta manera la cultura a las mujeres, ofreciéndoles también una educación que las preparaba profesionalmente. Es un lugar clave en donde se reúnen asociaciones de mujeres y entidades que llevan adelante actividades sobre feminismo, migración y ciudadanía. También allí, producto de las luchas de las mujeres migrantes y activistas como Norma Falconi y de la Asociación Papeles para tod@s, se comenzó a ocupar el espacio activamente.

Se podría concluir que el Centro Cultural Francesca Bonnemaison es un lugar significativo en sí mismo, en primer lugar por las luchas que se han llevado a cabo por muchas mujeres dentro de este espacio y en segundo lugar porque uno de los ejes de su funcionamiento tiene que ver con la migración. Pero en este caso, las activistas no son objeto de intervención sino que se encuentran interviniendo el espacio y apropiándose de él con una propuesta concreta.

En este andamiaje sentía que la práctica estaba nombrando la teoría, donde somos responsables de los mundos que se construyen de acuerdo a un posicionamiento histórico, donde existe la posibilidad de 
crear conocimientos encarnados desde una mirada especifica y particular a través de las conexiones y conversaciones compartidas, (Haraway, 1995/ 1991). Por tanto, la metodología emergía como parte de un proceso que implica vivir para transformar(nos), sentir para vibrar con otras y escribir(nos), para recordar(nos).

\section{Una apuesta hacia la investigación activista feminista}

La apuesta por desarrollar una investigación activista feminista terminó de dar forma a una manera de hacer y participar dentro del sindicato. El punto de partida está relacionado con cuestionar los dilemas duales de la ciencia en particular, la preocupación positivista de situar con objetividad e imparcialidad los fenómenos que se estudian, como la escisión investigadora/investigada, sujeto/objeto. Así, la primera interrogante que se me planteaba fue si era posible abordar y producir conocimiento desmarcándonos de una visión fragmentada de la ciencia, las personas y lo social.

Sin dejar de tener en cuenta que también nosotras nos encontramos socializadas en formas de aprendizaje e investigación que se amparan bajo el paraguas positivista y allí se encuentra el reto, tanto en ser conscientes de ellas como en devenir otras subjetividades en el propio proceso de investigación.

La siguiente interrogante que orientó este recorrido, fue cómo opera la interacción entre el espacio académico y el militante/activista. Algunas producciones (Colectivo Situaciones, 2004; Precarias a la Deriva, 2004) hacen referencia a la articulación académico-militante a través de la co-producción de conocimiento orientado a la acción. Otras en América Latina entre 1960 y 1970, planteaban una corriente amplia de pensamiento en la que confluyeron la Educación Popular, la Teología de la Liberación, la Comunicación Alternativa, la Investigación Acción Participativa y la Filosofia de la Liberación (Torres, 2007).

En este sentido, Rahman y Fals Borda (1989) cuestionaron las bases epistemológicas de los estudios comunitarios, sugiriendo que debían generarse desde una perspectiva crítica en donde "es necesario descubrir esa base para entender los vínculos que existen entre el desarrollo del pensamiento científico, el contexto cultural y la estructura de poder de la sociedad" (1989, p. 72). Es decir que la IAP no era tan sólo una metodología de investigación sino también la expresión del activismo social. 
Desde esta perspectiva algunas y algunos autores contemporáneos han trabajado difractando estos conceptos dentro de la Psicología Social y Comunitaria como los trabajos de Marisela Montenegro (2001), Karla Montenegro (2005), Antar Martínez (2009), y Alejandra de León (2010).

En el planteamiento de la propuesta realizada al sindicato, partí de las consideraciones mencionadas que luego derivaron en la posibilidad de realizar una IACF (Biglia, 2005). Pero, ¿qué entendemos por esta metodología? La IACF hace referencia a que no solo la situación investigada y las técnicas utilizadas son elementos importantes y no neutrales, sino que estos elementos deberán estar en todo el proceso de investigación y no solo en algunas de sus fases (Biglia, 2005).

Además, se plantean una serie de supuestos para seguir una investigación activista, feminista que hacen referencia a: 1) el compromiso para el cambio social; 2) la ruptura de la dicotomía publico/privado; 3) la relación interdependiente entre teoría y práctica; 4) el reconocimiento de una perspectiva situada; 5) la asunción de responsabilidades; 6) la valoración y el respeto de las agencias de todas las subjetividades; 7) la puesta en juego de las dinámicas de poder que intervienen en el proceso; 8) una continúa abertura a ser modificadas por el proceso en curso; 9) la reflexividad; 10) lógicas no propietarias del saber; y 11) la redefinición del proceso de validación del conocimiento utilizando diferentes métodos. ${ }^{8}$

Este endecálogo es tan solo una orientación, no son supuestos inmutables o verdades a seguir, es decir que son un punto de partida desde dónde comenzar a construir la investigación en su contexto específico, en donde se entretejen algunos puntos de enclave que pueden ser revisitados a lo largo de todo el proceso. Dicho escenario posibilita romper con el papel de observador participante, no participante, etnógrafo o experto que viene a decirnos quiénes somos. Uno es-parte-de, es decir que nos sumamos a las luchas y resistencias como una más que amplían las posibilidades de hacer juntos en la defensa del espacios y territorios para la vida (Botero, 2012).

Esto implicó trazar un mapa e insertarme en diversas lecturas de teóricas y activistas con relación a la participación de las mujeres en el movimiento obrero y sindical, así como su conexión con el movimiento feminista y el trabajo del hogar, las lecturas del blog de Sindihogar, la asistencia a charlas y jornadas vinculadas a la temática, conversaciones

8 Para una mayor ampliación del endecálogo propuesto, remitirse a Biglia (2005). 
informales con algunas activistas para el intercambio de intereses y desarrollo del trabajo y participación en la red de mails en la cual me incluyeron (en el sindicato) para interactuar también en el plano virtual. Construyendo un campo-tema (Spink, 2003) vivo al habitar Sindihogar en donde compartimos con algunas investigaciones activistas (Callén et al., 2007) que al ocupar este espacio-tiempo a través de diversas prácticas y experiencias de la actividad diaria, nos convertimos en un elemento más en tensión, participamos de ella, la constituimos y esta a su vez nos constituye performativamente como investigadoras.

Las interacciones en el espacio-tiempo también suponen una serie de efectos, tanto en nuestro trabajo académico como en las relaciones establecidas dentro del sindicato. Es decir, los tiempos disímiles entre ambos espacios, diferentes formas de trabajo y de pensar. Efectos, que posibilitan interrogar y ser interrogadas y a partir de allí, construir saberes junto a otras, conversando, pasando tiempo juntas, conviviendo, debatiendo y actuando (León, 2010). Con esto me refiero, por ejemplo cuando como investigadoras, tenemos que tomar determinadas decisiones con respecto a dónde van a ser publicados nuestros trabajos, con qué accesos y para quién, como también el consenso dentro del colectivo de lo que se quiere re-producir y lo que no.

Este espiral de reflexión, creación, acción me llevo a proponer un espacio dentro de una asamblea para discutir este texto, que ya había circulado vía mail por Sindihogar como por la revisión de pares para su publicación en esta revista, entendiendo que no haya actrices sociales que puedan conocer mejor sino que hay diferencias que posibilitan las conexiones.

Lo interesante fueron los comentarios realizados por ambas partes y su aproximación con respecto a la forma del discurso. Por lo cual, en la re-lectura de este texto hay una intención de vehiculizar la experiencia a través del diálogo sin que este se presente enajenado o separado de quien escribe y sin tanta "verborragia de teorias y autoras" (L. activista, en CC. 28/09/13) detallando los pasos que dimos en el diseño y puesta en práctica de la formación. Busca además poder constituirse en una forma de "defender a muerte otras formas de trabajar desde la base y que estas se reconozcan como válidas" (N, activista, en CC. 04/11/13) donde el interventor o inventora, el investigador o investigadora conforman una de las múltiples posiciones de sujeto que se implica en articulaciones en las que se define qué es lo problemático y cuáles son las posibles formas de transformación social (Montenegro y Pujo, 2003). 
En este sentido entendemos que la IACF se encuentra nutrida por una visión harawariana, donde el conocer se produce a partir de conexiones parciales entre posiciones materiales y semióticas donde existe una relación inmanente entre la posición del conocimiento y el conocimiento generado (Montenegro y Pujol, 2003). De alguna, manera aceptar que esta perspectiva es parcial y que por tanto tiene que ser sometida a consenso y críticas es lo que surge de comprender los conocimientos situados, tomando distancia de la "objetividad fuerte" (Harding, 1993) en que la condición de opresión (sujeto obrero, sujeto feminista) es razón suficiente para lograr un mejor conocimiento de la realidad y el peligro de caer en el esencialismo inherente al conocimiento desde los márgenes o desde el privilegio epistémico (Haraway, 1995/1991).

Estas palabras nos permiten comprender que el potenciar está en los encuentros encarnados que nos permiten desmarcarnos del realismo y del relativismo (Haraway, 1995/1991) y posicionarnos desde lo político entre el espacio académico y activista.

Esta posición surge de una experiencia vivida por las investigadoras (nosotras) que figura marcos de interpretación que desbordan al que llega como experto a formular y diagnosticar, pues los argumentos que se encuentran más acá del intelecto atraviesan el pensamiento, desde el cuerpo, la sangre y el espíritu (Botero, 2012). Esto nos resitúa para reflexionar en torno a las diferentes diferencias que cruzan nuestras autobiografias y la de otras/otros, recordándonos nuestra posición dentro de la investigación y/o, limitaciones y permitiéndonos caminar con ellas. Estas diferencias nos enriquecen y en el respeto de ellas nos mancomunamos.

\section{Discusiones: potencialidades y límites de la propuesta formativa a través del prisma de la IACF}

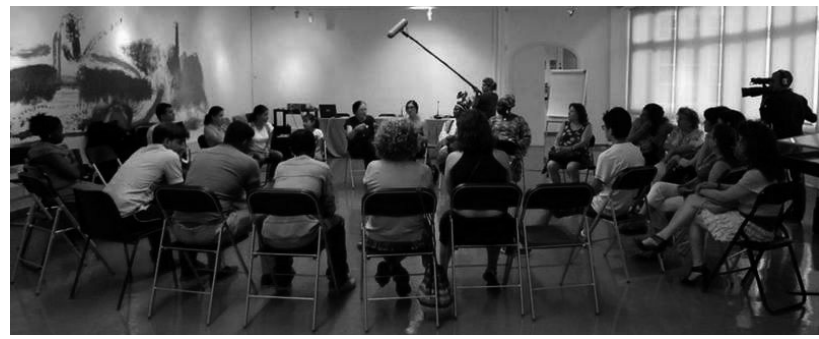

Foto: Jornadas "Las diferentes violencias". Norma Falconi (29/06/2013) 
En este apartado se comparten las reflexiones que surgieron tanto en el proceso como luego de las jornadas formativas, estas se entrelazan con los supuestos, potencias y límites que implica la IACF. En este sentido, proponer espacios entre la academia y el activismo es una posibilidad para discutir y comprender conjuntamente el trabajo realizado y que particularmente me llevó a aproximarme a nuevas formas de hacer, planteando algunas reflexiones al respecto.

\section{Potencias del proceso (IACF). De la apertura y flexibilidad}

"Sindihogar, con sus puertas abiertas a la formación para mujeres inmigrantes y con sus estrategias de captación ha creado un movimiento con vida, dinámico y ha tomado fuerza, todo un éxito para nosotras" (Activista, Casas, 2013, p.10). Estas palabras inscriben la pluralidad tanto de las activistas como de las participantes reivindicando su posicionamiento político que se corresponde con la visión de su conformación al invitar a la afiliación a mujeres y hombres que trabajen en hogares, por horas o de internas, que realicen cualquier tipo de trabajo relacionado con la limpieza y el cuidado. De igual forma pueden afiliarse tanto españoles o extranjeros que viven y trabajan en Cataluña y que tengan o no papeles, como aquellas personas que deseen apoyar al sindicato.

Estas luchas tienen sinergias con otras, donde las trabajadoras también han planteado la necesidad que se escuche lo que dicen las mujeres explotadas en este mercado laboral y se apoye realmente sus luchas, que nos atañen a todas y cuyas dimensiones norte-sur y migratorias son hoy muy importantes (Rodríguez, 2005). Es decir, un espacio donde quepan todas y se puedan discutir las problemáticas cotidianas del trabajo del hogar como una de las actividades laborales que la evolución del patriarcado y del capitalismo internacional desarrolla para que no se asuman las corresponsabilidades de los mismos.

En este sentido tengo que agradecer a Sindihogar por la formación brindada porque esto ha traído conmigo un cambio profundo y sobre todo ha establecido un compromiso de lucha y empoderamiento en mi personalidad que quiero trasmitir a mis compañeras. Desgraciadamente una mujer si se establece en la lucha de un objetivo sola, no tendrá la fuerza ni el impacto, como cuando muchas mujeres luchan por una misma causa, y el organizarnos como sindicato ha repercutido y le ha dado valor a nuestra institución. (Activista, Casas, 2013, p.9) 
De alguna manera, esto implica generar alianzas que tienen que ver más con el hacer que con lo que somos, a partir de construir lo que nos afecta, a través de una práctica compartida con otras, donde nuestra subjetividad también es modificada al compartir espacio-tiempo de las y los académicos, colectivos y activistas. Por otro lado supone la transformación que posibilita singular y colectivamente compartir y participar de estos espacios.

Este espíritu parte de dos consideraciones realizadas desde la $\mathrm{IACF}$, uno con respecto a la invitación de una continúa abertura a ser modificadas por el proceso en curso:

Partimos de la idea que es dentro de la ecuación espacio-tiempo, del contexto particular y situado, donde surge la posibilidad de comprender su dinámica y que para performar la transformación social es necesario tener en cuenta el micro-contexto continuamente, a diferencia de pensar sobre la trasformación porque entonces el espacio-tiempo actual no es tan importante. (Pujal, 2003, p.135)

El otro punto se refiere a "la intención explícita de que la investigación sea parte de un proceso para el cambio social en contra de las discriminaciones y/o los abusos de poderes" (Biglia, 2005, p.74), donde la potencia se encuentra en una apuesta al cambio y transformación como producto del mismo proceso.

En este sentido, no parece menos importante destacar la afección incluida en el conocimiento en tanto nos afectamos con otras y podemos comenzar un trabajo desde allí. El cruce se produce en el escenario de la vida cotidiana, donde nuestra subjetividad como investigadoras y como parte de un colectivo donde estamos colaborando (en mi caso, como parte del sindicato) se entrelazan, en tanto quien investiga acaba por involucrarse afectivamente con las personas con quienes realiza la investigación y esto se traduce en las propuestas, formas de trabajo y transformaciones individuales como colectivas.

\section{El respeto y la responsabilidad a la hora de trabajar en colectivo}

En este sentido una de las activistas hablaba de la "flexibilidad y apertura" (N. activista, en CC. 06/07/2013) haciendo referencia al poder acomodarse a los tiempos y responsabilidades que tienen fuera del colectivo e ir encontrando los huecos y lugares para trabajar juntas. 
Ahora que esta palabra esta "moda" [empoderamiento] también me sentí empoderada y me resultó fácil trabajar con la compañera que colaboro en la organización de la formación porque planteamos una metodología abierta y flexible. (N, activista, en CC. 06/07/2013)

Por un lado, se plantea una crítica a modo de ironia, interpelando las formas que existen de intervención donde se pretende empoderar a una o un otro y por otro lado, también se deja traducir la posibilidad de crear formas de trabajo donde todas pudiéramos ser actrices del proceso.

Esta forma de trabajo también me supuso particularmente, acomodarme a otros ritmos y saberes, sobre todo al trabajar conjuntamente con una activista con gran experiencia y comprometida en su hacer que compartió su pasión y conocimientos, contagiándome y contagiándonos, haciéndonos participes a todas.

De esta manera, se genera la valoración y el respecto de la agencia de todas las subjetividades (Biglia, 2005) lo cual también implica respetar los tiempos y lugares de cada una, sus recursos, recorridos y trayectorias. Además implica no ser ciegas a las relaciones de poder existentes, tanto dentro del colectivo y en mi caso, en tanto parte del mismo como investigadora, partiendo de la base, que las mismas no obstaculicen el trabajo, sino por el contrario que este pueda ser problematizado y asumido por todas.

\section{Potenciar las conexiones parciales}

El plantear un "taller interactivo y la disposición del espacio en forma de círculo" (N. activista en CC. 10/06/2013) hace referencia a la necesidad planteada por algunas activistas de generar un ambiente propicio para compartir conversaciones desde formas horizontales de participación y no solo de escucha, donde se pudiera dinamizar y circular la palabra. Esto nos permitió hablar de nuestras experiencias y conocer más aspectos de la ley. (R. activista en CC. 06/07/13). Es el círculo como mundo y diversidad en donde todas somos puntos de partida y de llegada en tanto cada una va tomando la palabra de acuerdo a las resonancias que se producen en lo dicho y en la narración de las experiencias.

Esto nos invita a reconocer con quién estamos y quién nos trastoca en este proceso de conocer (Araiza, 2009). Así, poder admitir por un lado un gozo en nuestro quehacer que podamos compartir con más 
gente, invitándonos a ponernos en riesgo, a "ser tocados y cambiemos, sin pretender que nosotros somos quienes escuchan y sin pretender que la comunicación perfecta realmente existe" (Araiza, 2009).

Al potenciar formas de trabajo horizontales impulsamos la posibilidad de generar conexiones parciales, es decir que se construyan en relación, como plantea León (2010), donde todas conocemos y somos conocidas, produciéndose una objetividad encarnada a través de las conversaciones y diálogos que mantenemos, responsabilizándonos de los efectos que se produzcan. Por ejemplo, al debatir lo que para cada una de las que nos encontrábamos en las jornadas significaba "violencia de género", nos llevó a visualizar las diferentes posturas que teníamos respecto a la misma. Para algunas esto significaba una violencia estructural (N. activista, en CC, 29/06/13), otra lo planteaba en términos de acoso laboral (M. activista, en CC, 29/06/13) y sexual (G. activista, en CC, 29/06/13), generándose así diversos discursos que se articularon en una puesta en común respecto a las estrategias para cuidarnos y prevenirlas (Montserrat, ponente, en CC, 29/06/13).

De esta manera nos permitimos romper con roles estáticos, habilitando encontrarnos a veces como participante, ponente, colaboradora, lo que implica asumir que nuestra cotidianidad está ocupada de múltiples identidades, lo cual no puede desprenderse de nuestro quehacer como investigadoras, trabajadoras y/o activistas. Sin embargo, no dejamos de reconocer nuestra propia a-neutralidad y de las propias responsabilidades con relación a lo producido, los logros, como a todo el proceso, en el que están en juego nuestras propias elecciones más que los roles sociales que puedan estar determinados (Biglia, 2005).

\section{Re-conocernos como un enclave de la práctica feminista}

Las temáticas que se abordaron, sobre todo las normativas nacionales como internacionales respecto al trabajo del hogar y de la ley de extranjería, posibilitaron no solo repetir todos los puntos de las leyes, sino poder interpretarla y "discutir cómo a cada una afecta en su experiencia singular" (M. activista en CC. 01/06/13).

\footnotetext{
"Let's dismystify research, anyone can do it. Let's look away of being listened, let's put ourselves in risk of being touched and change without pretending we are ones of those who are listening, without pretending the perfect communication actually exists" (Seminario del MACBA, mayo 2006, en Araiza, 2009).
} 
Por otro lado, también se mencionó la "importancia de hacer un recorrido por lo que fue la lucha de las mujeres en la segunda república, recordando otras luchas" (N. activista en CC. 06/07/2013). En este sentido se trata de rescatar la importancia de partir de una genealogía de los movimientos para recordar los legados de las mujeres que nos antecedieron, tal como subrayan las prácticas feministas (Roseneil, 2000) y por tanto, poder considerar la importancia de mencionar los orígenes y las raíces que han hecho posible la constitución, tanto de este colectivo como de otros (Biglia, Bonet y Martí, 2006).

Esto también nos permite hacer un trazo entre rupturas y continuidades que se producen dentro de los movimientos, donde según Biglia (2005) hay una tendencia que lleva a definir las nuevas olas de activismo social como separadas de las precedentes organizaciones.

\section{Limites de la propuesta (IACF). Los desafios en el lenguaje}

Algunas de las limitantes o desafios que se plantearon, en torno a la experiencia tuvieron que ver en primer lugar, con la interpelación de las activistas respecto al lenguaje académico o técnico utilizado en algunas ocasiones. Esto ocasionaba una interferencia o ruido en la comunicación que es necesario trascender para poder ser inteligible entre nosotras; además de la pluralidad idiomática y cultural que tiene como característica el sindicato, e hizo necesario la participación de compañeras que pudieran hacer de traductoras, poniéndonos en juego para no caer en una universalidad occidental hegemónica que anula la diversidad, buscando utilizar herramientas y recursos que nos permitan re-encontrarnos en estos espacios.

\section{Trascender nuestros espacios públicos/privados}

Por otro lado también se planteó que, si bien existió una convivencia en el espacio con los niños que acompañaron a las compañeras en las actividades y tenían su lugar para sumarse a las actividades artísticas, participando de las performance, la danza y la plástica, una activista propuso "la posibilidad de tener a futuro una monitora para estas actividades" (N, activista, en CC. 06/07/13) posibilitando trascender la ruptura dicotómica de nuestros espacios públicos/ privados, siguiendo la orientación metodológica de la IACF, invitándonos a participar y ser parte de este espacio en nuestro entorno (amigas(os), músicos, bailarinas, etc.). 


\section{Sobre los formatos}

Por último pienso en todo lo que ha quedado por fuera de este formato de escritura (la performance, las cartas realizadas para enviar a los CIE's, la música, el canto, los videos productos de las jornadas...) que nos permitió sostener un espacio de reflexión y disfrute simultáneamente, que a la distancia leo como una práctica corporal de libertad y resistencia, como una forma de subvertir la hostilidad que se presenta en la cotidianidad. Estos otros recorridos quedan en la experiencia y en el cuerpo y son de dificil traducción en formatos académicos, que muchas veces encorsetan nuestras formas de hacer. En este sentido, la IACF es una apuesta por problematizar y acercarnos a cierta reflexividad a partir de lo que hacemos -que no implican necesariamente un perfeccionamiento sino poner en evidencia sus características y limitaciones-, lo cual nos permite continuar abiertas al cambio y plantear los límites y/o tensiones específicos y contextuales en cada proceso de investigación. Es decir, que esto también nos posibilita salir de posiciones culposas por encontrarnos en los entre para posicionarnos desde las contradicciones que trascienden las propias investigaciones, ya que estas mismas emergen como parte de nuestros procesos vitales.

\section{Algunos apuntes finales en torno a la IACF}

La investigación activista se encuentra atravesada por la construcción de memorias y narrativas colectivas en contextos próximos donde nos articulamos con una práctica de reflexión-creación-acción. A la distancia la experiencia de la formación deja entrever la espiral que fue parte de lo que -sin saber con exactitud- estábamos haciendo al plantearnos estas jornadas.

A través de las opciones ontológicas y epistémicas optadas, esta investigación también pretende dialogar sobre las formas de producir conocimiento. Por lo cual la opción de una investigación activista feminista es una apuesta politica para re-pensar nuestros marcos metodológicos hacia lugares no propietarios del conocimiento y falsas dicotomías. Así, dentro de este proceso no solo se ha decidido la publicación del texto en una revista de acceso abierto, sino que -a partir del trabajo colectivo con las activistas- hemos publicado una revista próxima a los movimientos sociales, fusionándose la práctica política y la producción de conocimiento. 
La investigación y el activismo están relacionados a partir del reconocimiento de que lo personal es político, encontrando allí su enclave feminista ya que no se propone que las investigaciones distingan entre espacios públicos y privados, entre sujetos y objetos, razón y emoción y otros dualismos, sino que estos están interrelacionados y se influencian de manera continua.

Por otro lado, también es una invitación a conocer aquello que se encuentra más próximo en nuestra cotidianidad, cuestionando un pensamiento único y universal que no considera sistemas de opresión articulados como son el sexismo, el racismo, la heterosexualidad obligatoria, el clasismo y el neoliberalismo. En este sentido, la propuesta de la IACF -tanto en su apuesta política como ética de la responsabilidad en el conocimiento- es una posibilidad de generar disidencias temáticas a través de elaborar críticas a injusticias normalizadas (racismo, patriarcado, exclusión); así como disidencias metodológicas a través de investigaciones posicionadas, horizontales y participativas.

Si bien hemos propuesto algunos límites a partir de la puesta en práctica de la IACF contextualizada en esta experiencia, no podemos dejar de considerar que desde los movimientos, las investigaciones promovidas por activistas que se ubican en la academia pueden percibirse como ajenas y con cierta desconfianza, entendiendo que se reduce la capacidad de producción intelectiva de los movimientos sociales con el fin de obtener una legitimación académica. (Pantera Rosa, 2004).

En este sentido se propuso un método guiado por el "trueque constructivo" (León, 2010) y tiene como núcleo colaborar sin estorbar, trabajar por intercambio y acompañar a fortalecer la red afectiva de los colectivos hasta donde estos juzguen pertinente, desde posiciones performativas más que observadoras.

Por último, remarcar que "[...] sabemos ya que nuestras mejores ideas son producto del trabajo y del pensamiento conjunto" (Alexander y Mohanty, 2004/1997, p.138) y por tanto este texto cobró vida, en el ir y venir de las diferentes re-lecturas de las activistas, académicas, compañeras, amigas, convirtiéndolo en un texto mutado con la posibilidad de abrir otros campos de discusión. 


\section{Referencias}

Adán, C. (2006). Feminismo y conocimiento. De la experiencia de las mujeres al cíborg. La Coruña: Spiralia.

Alexander M., y Mohanty. C. (2004/1997). Genealogias, legados, movimientos En b., hooks, A., Brah et al. Otras inapropiables, Feminismos desde las fronteras. (pp.137-184) Madrid: Traficantes de sueños.

Araiza, A. (2009). La propuesta de Donna Haraway, en Conocer y ser a través de la práctica del yoga: una propuesta feminista de investigación preformativa. Tesis no publicada de doctorado en Psicología Social. Universidad Autónoma, Barcelona, España.

Balasch, M. y Montenegro, M. (2003). Una propuesta metodológica desde la epistemología de los conocimientos situados: Las producciones narrativas. Encuentros en Psicología Social, 1 (3), 44 - 48

Biglia, B. (2005). Narrativas de mujeres sobre las relaciones de género en los movimientos sociales. Tesis Doctoral no publicada. Departamento de Psicologia Básica. Universidad de Barcelona, España.

Biglia, B., Bonet, J., y Marti, M. (2006). Experiencias y reflexiones de investigacció en allo ciutadania i recerca a la Universitat. Disponible en: http:/ / www.academia.edu/313073/Experiencias_Y_Reflexiones_De_Investigaccio

Biglia, B. y Zavos. (2005). Situar-nos a dins, a fora o a la frontera. Quines (im) posibles relacions entre l'activisme i l'acadèmia en les "investigacions crítiques". En Investigacció, Moviments socials i investigació activista (pp. 83-90). Barcelona: E1 Viejo Topo.

Botero, P. (2012). Investigación y acción colectiva -IAC- Una experiencia de investigación militante. Utopía y Praxis Latinoamericana, 17 (57), 31-47.

Callén, Balasch, Guarderas, Gutierrez, León, Montenegro, Montenegro, y Pujol, (2007). Apuntes epistemo-politicos desde una etnografia tecnoactivista [41 párrafos]. Forum Qualitative Sozialforschung / Forum: Qualitative Social Research, 8(3). Riereta.net.

Casas, A. (2013). Informe Bilbao Junio 2013, Sindihogar. Manuscrito no publicado.

Colectivo Situaciones (2004). Algo más sobre la militancia de investigación. Notas al pie sobre procedimientos e (in)decisiones. En Malo, M. Nociones comunes. Experiencias y ensayos entre investigación y militancia (pp. 93-110). Madrid: Traficantes de Sueños.

Collins Hill, P. (2012/2000). Rasgos distintivos del pensamiento feminista negro. En Jabardo, M. (Ed.) Feminismos negros. Una antología (pp. 99-131). Madrid: Mercedes Jabardo y Traficantes de Sueños.

Grimberg, M. (2009). Poder, política y vida cotidiana: un estudio antropológico sobre protesta y resistencia social en el área metropolitana de Buenos Aires. 
Revista de Sociológica e Política, 17 (32). Disponible en: http://www.scielo.br/ pdf/rsocp/v17n32/v17n32a06.pdf

Fals Borda, O. (1993). La investigación participativa y la intervención social. Documento Social, 92, 9-22.

Harding, S. (1993). Ciencia y feminismo. Madrid: Morata.

Haraway, D. (1995/1991] Ciencia, cyborgs y mujeres. La reinvención de la naturaleza. Madrid: Ediciones Cátedra S.A.

IOE. (Colectivo) (1993). AP. Introducción en España, Documentación Social, 92, 59-69.

León, A. (2010). Danzando la psicología social comunitaria: revisitando la IAP a partir de un curso de danza en una asociación cultural de barrio. Athenea Digital, 17, 255-270.

Lewin, K. (1946). Action-research and minority problems. Journal of Social Issues, 2, 34-46.

Longino, H. (1990). Science as Social Knowledge: Values and Objectivity in Scientific Inquiry. Princeton: Princeton University Press.

Martínez,, A. (2009). Movimientos adyacentes para re-pensar la InvestigaciónAcción Participante. Disponible en: http://psicologiasocial.uab.es/fic/ca/ book/2009/02/16

Montenegro, M. (2001). Conocimientos, agentes y articulaciones: una mirada situada a la intervención social. Tesis de Doctorado en Psicología Social. Bellaterra: Universidad Autónoma de Barcelona, España.

Montenegro, M. y Pujol, J. (2003). Conocimiento Situado: Un Forcejeo entre el Relativismo Construccionista y la Necesidad de Fundamentar la Acción. Revista Interamericana de Psicologia/Interamerican Journal of Psychology, 37 (2), 295-307.

Montenegro, K. (2005). La intervención social sobre el desarrollo. Reflexiones desde la actividad interventora en Nicaragua. Tesis de Maestría en Psicología Social. Bellaterra: Universidad Autónoma de Barcelona, España.

Pantera, R. (2004). Moverse en la incertidumbre. Dudas y contradicciones de la investigación activista. En Malo, M. Nociones comunes. Experiencias y ensayos entre investigación y militancia (pp. 191-205). Madrid: Traficantes de Sueños.

Precarias a la deriva (2004). A la deriva por los circuitos de la precariedad femenina. Madrid: Traficantes de Sueños.

Pujal, M. (2003). La tarea crítica: interconexiones entre lenguaje, deseo y subjetividad. Politica y Sociedad, 40 (1), 129-140

Rodríguez, C. (2005). Feminismos disidentes en América Latina y el Caribe. Nouvelles Questions Feministes, 24 (2), 101-105. 
Rahman, A. y Fals Borda, O. (1989). La situación actual y las perspectivas de la IAP en el mundo. Análisis Politico, 5, 14-20. Disponible en: http://gumilla. org/biblioteca/bases/biblo/texto/COM199694_14-20.pdf

Roseneil, S. (2000). Common women, uncommon practices. The queer feminism of greenham. London and New York: Cassell.

Spink, P. (2003). Pesquisa de campo em psicolgia social: uma perspectiva pósconstrucionista. Psicologia \& Sociedade, 15 (2), 18-42. Disponible en: http:// www.scielo.br/pdf/psoc/v15n2/a03v15n2.pdf

Torres, A. (2007). La educación popular. Trayectorias y actualidades. Bogotá: El Búho.

Zavos, A., Biglia, B., y Hoofd, I. (2005). Questioning the Political Implications of Feminist Activism and Research in Different Settings. CASAzine, 1, 4-6.

\section{Cómo citar este artículo}

Fulladosa-Leal, K. (2015). Creando puentes entre la formación y la creatividad: Una experiencia de investigación activista feminista. Universitas Humanística, 79, 115-140. http://dx.doi.org/10.11144/Javeriana.UH79.cpfc 